\title{
Divulgación de información responsable por los gobiernos locales españoles
}

\author{
María Pache-Durán* \\ María Teresa Nevado-Gil*
}

Artículo recibido:

23 de octubre de 2018

Artículo aceptado:

19 de marzo de 2019

Artículo de investigación

\section{Resumen}

El acceso a la información pública, la transparencia y las normas de buen gobierno son los ejes fundamentales de cualquier ente público en el siglo XXI. El objetivo del presente estudio consiste en analizar la información sobre responsabilidad social que ofrecen las 50 capitales de provincias españolas a través de sus sitios web, utilizando la técnica de análisis de contenido. Los resultados muestran que la información más divulgada está relacionada con la contratación de servicios y obras públicas, en contraposición a la de carácter medioambiental, que es la menos difundida. A pesar de ello, a los gobiernos locales españoles aún les

* $\quad$ Facultad de Empresa, Finanzas y Turismo, Universidad de Extremadura, España mpache@unex.es tnevado@unex.es

INVESTIGACIÓN BIBLIOTECOLÓGICA, vol. 33, núm. 81, octubre/diciembre, 2019, México, ISSN: 2448-8321 pp. 111-134 
queda un largo camino por recorrer en materia de divulgación de información responsable.

Palabras clave: Divulgación de Información; Responsabilidad Social; Gobiernos Locales; Sitios Web

\title{
Disclosure of responsible information by Spanish local governments
}

María Pache-Durán and María Teresa Nevado-Gil

\begin{abstract}
Access to public information, transparency and standards of good governance are the cornerstones of any public entity in the 21st century. The objective of the present study is to analyse the information on Social Responsibility offered by the 50 capitals of Spanish provinces through their websites and the use of content analysis. In addition, the possible similarities and differences between local governments regarding these levels of disclosure are also analysed. Results show that the most popular information is related to the contracting of services and public works, as opposed to the environmental one, which is the least disseminated. Despite this, Spanish local governments still have a long way to go in terms of disseminating responsible information.
\end{abstract}

Keywords: Disclosure; Social Responsibility; Local Government; Websites.

\section{INTRODUCCIÓN}

$E^{1}$ constante cambio en el que se sumerge la sociedad en la primera mitad del siglo XXI ha ocasionado la aparición de distintos enfoques en lo que respecta a la responsabilidad social (RS), lo que ha supuesto una confusión terminológica derivada de que cada investigador asume la RS desde su propia perspectiva (Valor y Hurtado, 2009). Aunque algunos consideran que el problema principal radica en que estamos ante una teoría relativamente nueva (Mullerat, 2010), autores como Freeman et al. (2010) argumentan que el 
problema proviene del uso que se está haciendo del mismo, lo que provoca la pérdida de su significado.

La RS es un concepto amplio y complejo y, aunque hay múltiples definiciones, no hay una clara unanimidad debido a la variedad de temas que aborda (Kolk, 2010). A pesar de su importancia aún carece de una definición generalmente aceptada (Green y Peloza, 2011), por lo que ha de ser entendida como un proceso de integración y mejora continua que busca conciliar el crecimiento y la competitividad combinando a su vez un compromiso permanente con la mejora medioambiental y el desarrollo social. A pesar de encontrar una multitud de definiciones al respecto, el número de conceptualizaciones de RS ha disminuido en los últimos años (Kolk, 2016). A continuación, presentamos aquellas definiciones que se consideran más relevantes.

En un contexto internacional, la Comisión Europea, en su Libro Verde (COM, 2001: 4) y en su Comunicación 2002/347, define la RS como "la integración voluntaria, por parte de las empresas, de las preocupaciones sociales y medioambientales en sus operaciones comerciales y sus relaciones con sus interlocutores". Esta publicación supuso uno de los grandes hitos a favor de la RS. En España, el Foro de Expertos en RS constituido en el seno del Ministerio de Trabajo y Asuntos Sociales la define como

además del cumplimiento estricto de las obligaciones legales vigentes, la integración voluntaria en su gobierno y gestión, en su estrategia, políticas y procedimientos, de las preocupaciones sociales, laborales, ambientales y de respeto a los derechos humanos, que surgen de la relación y el diálogo transparentes con sus grupos de interés, responsabilizándose así de las consecuencias y los impactos que derivasen de sus acciones. (Ministerio de Trabajo y Asuntos Sociales, 2005: 7)

Por su parte, la Asociación Española de Contabilidad y Auditoría define la RS como "el compromiso voluntario de las empresas con el desarrollo de la sociedad y la preservación del medio ambiente, desde su composición social y un comportamiento responsable hacia las personas y grupos sociales con quienes se interactúa” (AECA, 2004: 21).

La importancia de la RS repercute del mismo modo en las organizaciones de las administraciones públicas, por lo que deben llevarse a cabo políticas públicas responsables plasmadas a través de la divulgación de la información, fomentando así su sostenibilidad y transparencia informativa (Nevado, Gallardo y Sánchez, 2013; Navarro, Alcaraz y Ortiz, 2016; López, 2010; López, 2006). La transparencia en la información pública es indispensable para la toma de decisiones y el pluralismo participativo, es decir, suministrar información al público y evitar acciones corruptas, entre otras funciones 
(Shuler, Jaeger y Bertot, 2010). A lo largo de la literatura podemos encontrar gran cantidad de estudios que analizan la importancia de la divulgación de información a través del uso de las Tecnologías de la Información y las Comunicaciones (TIC) en las administraciones públicas (Pacios, 2016; Garde, Rodríguez y López, 2015). En los últimos años, uno de los mecanismos de transparencia administrativa que ha ido alcanzando una importancia relevante gracias al uso de las TIC es la divulgación de información a través de los sitios web (Chaín, Muñoz y Más, 2008; Ayuso y Martínez, 2005). Esta nueva forma de interacción de los gobiernos locales con sus stakeholders, conocida como administración electrónica, e-administración, gobierno electrónico o e-gobierno, dará lugar a un gobierno más eficiente, responsable y transparente (García López, 2007; Bannister y Connolly, 2011).

Con respecto al ámbito local, la revisión de la literatura pone de manifiesto en los últimos años el incremento de estudios que se centran en el análisis de la información divulgada, ya sea voluntaria, financiera, sostenible o de RS. Centrándonos en estos dos últimos aspectos, encontramos estudios que analizan al grado de divulgación de información pública de los gobiernos locales. Así, en el ámbito nacional, encontramos trabajos como los de Navarro, Alcaraz y Ortiz (2010), que analizan los sitios web de 55 gobiernos locales, realizando una aproximación a la difusión de información sobre RS y la publicación de información económica, social y medioambiental; Prado-Lorenzo, García-Sánchez y Cuadrado-Ballesteros (2012), que analizan 78 municipios españoles de más de 100000 habitantes; García-Sánchez, Frías-Aceituno y Rodríguez-Domínguez (2013), que estudian los sitios web de 102 gobiernos locales españoles; Cuadrado-Ballesteros, Frías-Aceituno y Martínez-Ferrero (2014), que examinan 102 municipios españoles; Alcaraz-Quiles, Navarro-Galera y Ortiz-Rodríguez (2015), que se encargan de analizar 55 gobiernos locales españoles, y Nevado, Milanés y Prieto (2018), que estudian 213 municipios de la región de Cáceres.

Por otro lado, en el ámbito internacional, encontramos algunos autores como Frías-Aceituno, Marques y Rodríguez-Ariza (2013), que estudian el contenido de 25 sitios web de municipios brasileños, colombianos, mexicanos, portugueses y españoles; Moura, Ribeiro y Monteiro (2014), que se centran en la divulgación de información responsable en 60 municipios portugueses; Mir, Chatterjee y Taplin (2015), que se encargan de analizar la divulgación de información de 85 gobiernos locales de Nueva Zelanda; Navarro et al. (2015), que analizan los sitios web de 21 de los gobiernos locales de países nórdicos; Brusca, Manes Rossi y Aversano (2016), que examinan 46 gobiernos locales italianos y 59 españoles, y Hashim et al. (2016), que centran su muestra en 98 entidades locales en Malasia, entre otros. 


\section{Metodología}

El objetivo del presente estudio consiste en analizar la información sobre RS que ofrecen los 50 gobiernos locales que conforman las capitales de provincias españolas a través de sus sitios web, utilizando la técnica de análisis de contenido. De acuerdo a nuestro objetivo, nos hemos planteado las siguientes preguntas de investigación:

Q1. ¿Divulgan información responsable los gobiernos locales españoles a través de sus sitios web?

Q2. ¿Cuál es el grado de dicha información divulgada?

Q3. ¿Cuál es su naturaleza? Esto es, ¿`cuál es el tipo de información que divulgan? La respuesta a esta pregunta nos dará a conocer la im plicación y el compromiso de los gobiernos locales en relación a los diferentes aspectos relacionados con la RS.

La justificación de las preguntas de investigación que planteamos tiene su base en la importancia de la difusión de información pública a través de las páginas web como mecanismo de transparencia administrativa. En los últimos años se han publicado diferentes estudios relativos a la divulgación de información pública por parte de las administraciones públicas, motivado por un crecimiento significativo del interés del ciudadano hacia la transparencia de los gobiernos en materia de RS (Nevado, Gallardo y Sánchez, 2013). Por tanto, en este trabajo planteamos el estudio del análisis de la información divulgada y, concretamente, del grado y de la naturaleza de dicha información.

En relación a la muestra, se eligieron los gobiernos locales de las capitales de provincia de España que, como establece la sinopsis del artículo 141 de la Constitución Española, desde 1927 pasa a tener 50 capitales de provincia cuando Las Palmas de Gran Canaria se separa de Santa Cruz de Tenerife. Los 50 gobiernos locales seleccionados suman un total de 14833628 habitantes, resultantes de la revisión de los padrones municipales, según el RD 1039/2017, de 15 de diciembre, por el que se declaran oficiales las cifras de población resultantes de la revisión del padrón municipal referidas al 1 de enero de 2017. Se considera, por tanto, una muestra capaz de arrojar resultados significativos respecto al nivel de divulgación de información, puesto que se considera relevante estudiar hasta qué punto los gobiernos locales de las capitales de provincia están desarrollando una adecuada labor de difusión mediante el uso de las TIC para contribuir a la divulgación de información responsable.

Con el fin de alcanzar los objetivos de nuestro estudio, esta investigación parte de la compilación de datos relacionados con la divulgación de 
información en materia de RS a través de los sitios web de los gobiernos locales utilizando la técnica de análisis de contenido empleada en investigaciones previas (Navarro et al., 2011; Moneva y Martín, 2012; Nevado, Gallardo y Sánchez, 2013; Beuren y Angonese, 2015; García-Sánchez, Frías-Aceituno y Rodríguez-Domínguez, 2013). La recopilación de los datos se llevó a cabo a través del acceso mediante el buscador de la página principal de cada ente local entre los meses de enero y junio de 2018. El criterio utilizado para puntuar el nivel de divulgación sobre RS fue asignar a cada indicador una escala dicotómica, es decir, el valor 1 si el ítem es divulgado por el gobierno local estudiado y 0 en caso contrario (Nevado, Gallardo y Sánchez, 2016; Nevado y Gallardo, 2016; Frías-Aceituno, Marques y Rodríguez-Ariza, 2013, entre otros).

Para ello, se han utilizado los indicadores propuestos en estudios previos por Nevado, Gallardo y Sánchez (2013) compuestos por un total de 118 indicadores divididos en cinco ejes de análisis (Tabla 1). Debido a la desaparición de las financiaciones por parte del Fondo de Sostenibilidad hemos adaptado tales indicadores de tal forma que en total disponemos de 115. El resultado, por lo tanto, es el siguiente: el eje de análisis 1 contiene 27 indicadores relativos a información sobre la corporación municipal; el eje 2, 28 indicadores referentes a información social, sobre los ciudadanos y la sociedad; el eje 3 incluye 21 indicadores sobre información económica; el eje 4, 24 indicadores de información sobre contratación de servicios y obras públicas y, por último, el eje 5 , que contiene 15 indicadores sobre información medioambiental.

\begin{tabular}{|l|c|c|c|}
\hline \multicolumn{1}{|c|}{ Eje de análisis } & $\begin{array}{c}\text { N }{ }^{\mathbf{0}} \text { de } \\
\text { indicadores }\end{array}$ & Peso índice & Ponderación \\
\hline 1. Información sobre la corporación municipal & 27 & $23.48 \%$ & $20 \%$ \\
\hline $\begin{array}{l}\text { 2. Información sobre los ciudadanos y la } \\
\text { sociedad }\end{array}$ & 28 & $24.35 \%$ & $20 \%$ \\
\hline 3. Información económica & 21 & $18.26 \%$ & $20 \%$ \\
\hline $\begin{array}{l}\text { 4. Información sobre la contratación de } \\
\text { servicios y obras públicas }\end{array}$ & 24 & $20.87 \%$ & $20 \%$ \\
\hline 5. Información medioambiental & 15 & $13.04 \%$ & $20 \%$ \\
\hline \multicolumn{1}{|c|}{ Total } & 115 & $100 \%$ & $100 \%$ \\
\hline
\end{tabular}

Tabla 1. Indicadores por eje de análisis Fuente: adaptado de Nevado, Gallardo y Sánchez (2013; 2016)

Una vez analizados los sitios web objeto de estudio se elaboran los índices de divulgación partiendo de los propuestos por Nevado, Gallardo y Sánchez 
(2016) y Nevado y Gallardo (2016), y desarrollados por Carvalho, Gallardo y Nevado (2018), con el objetivo de analizar el grado y el tipo de información responsable que los gobiernos locales de las capitales de provincia españolas divulgan. La medición del nivel de información mediante índices se ha llevado a cabo en estudios como los de Navarro, Alcaraz y Ortiz (2010) y Navarro et al. (2015), Moneva y Martín (2012), Gandía y Archidona (2008), y Beuren y Angonese (2015), entre otros.

En este contexto, se realiza una medición de la divulgación de información en dos niveles. Por un lado, se mide la divulgación por gobierno local en cada uno de los ejes de análisis a partir de un índice de partida (IDGE) y, a continuación, se calcula un nuevo índice con el objetivo de medir la información total en cada gobierno (IDG). Por otro lado, se calcula la divulgación total de cada indicador (IDI), a partir del cual se construye otro para cada uno de los ejes de análisis (IDE) y se culmina con la medición de la divulgación total de la muestra (IDT) (Tabla 2).

\begin{tabular}{|c|c|c|}
\hline Índices & Concepto & Expresión \\
\hline $\begin{array}{l}\text { Índice de divulgación por } \\
\text { gobierno local y eje de } \\
\text { análisis (IDGE) }\end{array}$ & $\begin{array}{l}\text { Mide el porcentaje de } \\
\text { divulgación total de cada } \\
\text { gobierno local en cada uno } \\
\text { de los ejes de análisis }\end{array}$ & $\begin{array}{c}I D G E_{j}=\left(\frac{\sum_{i=1}^{M}(A i j)}{M} * p\right) \\
* 100\end{array}$ \\
\hline $\begin{array}{l}\text { Índice de divulgación por } \\
\text { gobierno local (IDG) }\end{array}$ & $\begin{array}{l}\text { Mide la divulgación total de } \\
\text { cada gobierno local }\end{array}$ & $I D G_{j}=\sum_{i=1}^{E}\left(I D G E_{j}\right)$ \\
\hline $\begin{array}{l}\text { Índice de divulgación por } \\
\text { ítem (IDI) }\end{array}$ & $\begin{array}{l}\text { Mide el porcentaje de } \\
\text { gobiernos locales que } \\
\text { informan cada ítem }\end{array}$ & $I D I_{i}=\frac{\sum_{i=1}^{N}(A i j)}{N} * 100$ \\
\hline $\begin{array}{c}\text { Índice de divulgación por eje } \\
\text { de análisis (IDE) }\end{array}$ & $\begin{array}{l}\text { Mide la divulgación total de } \\
\text { cada eje de análisis }\end{array}$ & $I D E_{t}=\left(\frac{\sum_{i=1}^{M}\left(I D I_{i}\right)}{M} * p\right)$ \\
\hline $\begin{array}{l}\text { Índice de divulgación total } \\
\text { (IDT) }\end{array}$ & $\begin{array}{c}\text { Mide la divulgación total de } \\
\text { la muestra }\end{array}$ & $I D T=\sum_{i=1}^{E}\left(I D E_{i}\right)$ \\
\hline
\end{tabular}

Tabla 2. Índices de divulgación

Fuente: adaptado de Carvalho, Gallardo y Nevado (2018)

$M=$ número de ítems que forman cada eje de análisis; $E$ = número de ejes de análisis; $A i j=$ toma el valor de 1 si la característica que define el indicador ( $i$ ) está presente en el gobierno local ( $j$ ) y 0 en el caso contrario; $N$ = número de gobiernos locales. Al no tener evidencias empíricas sobre la importancia de los distintos índices parciales que componen el índice total se ha asignado el mismo peso específico para cada una de las dimensiones $(p=20 \%)$ 
En el Anexo 1 se plasman los resultados obtenidos en los índices de divulgación de información por ítems (IDI). De manera desglosada y partiendo del primer eje de análisis, información sobre la corporación municipal, se puede observar en la Figura 1 que la mayoría de los gobiernos publica la relación de puestos de trabajo (98\%), la Agenda 21 local (98\%) y un "Código de buen gobierno" (98\%). Además, casi todos especifican los datos biográficos y direcciones electrónicas del Alcalde y los concejales $(97 \%$ ) y publican las Ordenanzas y Reglamentos Municipales (96\%). En contraposición a lo anterior, sólo $2 \%$ identifica los compromisos cumplidos del programa de gobierno e incluye eventos, logros y fracasos durante el periodo de información, y $17 \%$ incluye en su declaración los desafíos y metas para los próximos 3/5 años.

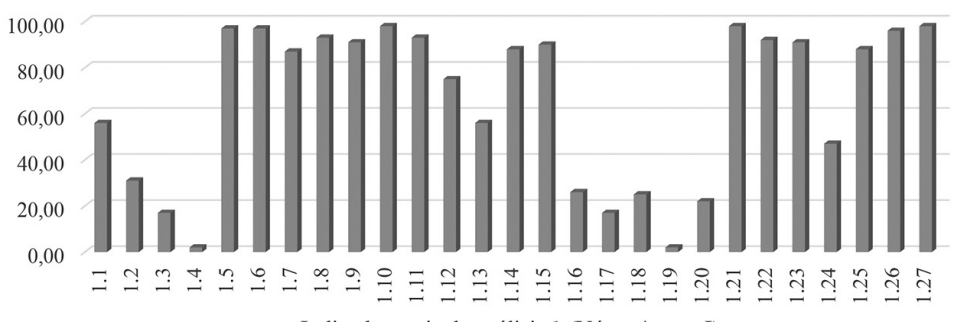

Indicadores eje de análisis 1 (Véase Anexo I)

Figura 1. Índices de divulgación de información de cada ítem en el eje 1 de análisis (IDI) Fuente: elaboración propia

En relación al segundo eje de análisis (Figura 2), información social (los ciudadanos y la sociedad), todos los sitios web de los gobiernos tienen un buscador interno en la página web, ofrecen la posibilidad de realizar trámites administrativos, permisos y licencias online, tienen un buzón ciudadano o una sección para quejas y sugerencias, radio municipal y publican noticias destacadas. Sin embargo, ninguna capital divulga el salario mínimo local, no tienen wikis compartidas entre departamentos ni webcams. Además, cabe destacar que $85 \%$ de los gobiernos ofrecen la posibilidad de aportar sugerencias y participación ciudadana en la elaboración de los presupuestos. Por contra, un porcentaje mínimo de sitios web dispone de un servicio de mensajería móvil (2\%) y de un servicio de chat (1\%). 


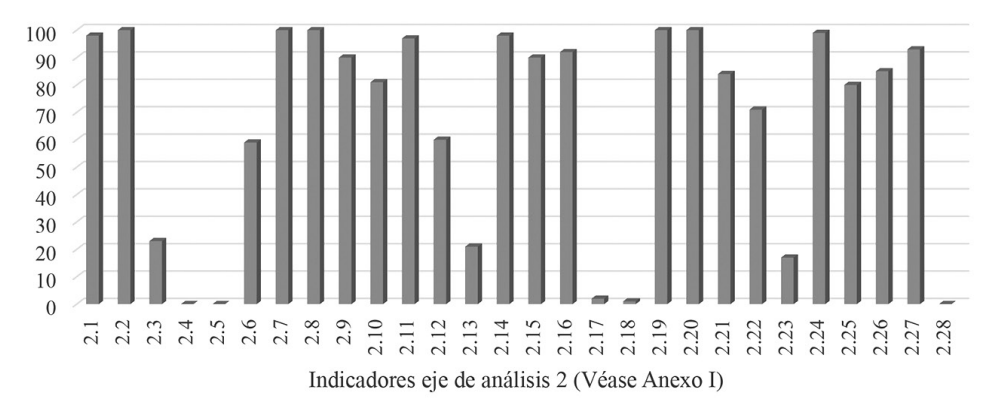

Figura 2. Índices de divulgación de información de cada ítem en el eje de análisis 2 (IDI) Fuente: elaboración propia

Del análisis del tercer eje, que corresponde a la información económica, se puede observar en la Figura 3 que $97 \%$ de las webs analizadas disponen de una publicación de modificaciones presupuestarias; $89 \%$ divulga sus cuentas anuales y $88 \%$ publica informes periódicos sobre la ejecución del presupuesto. Sin embargo, tan sólo 18 \% informa sobre el endeudamiento relativo.

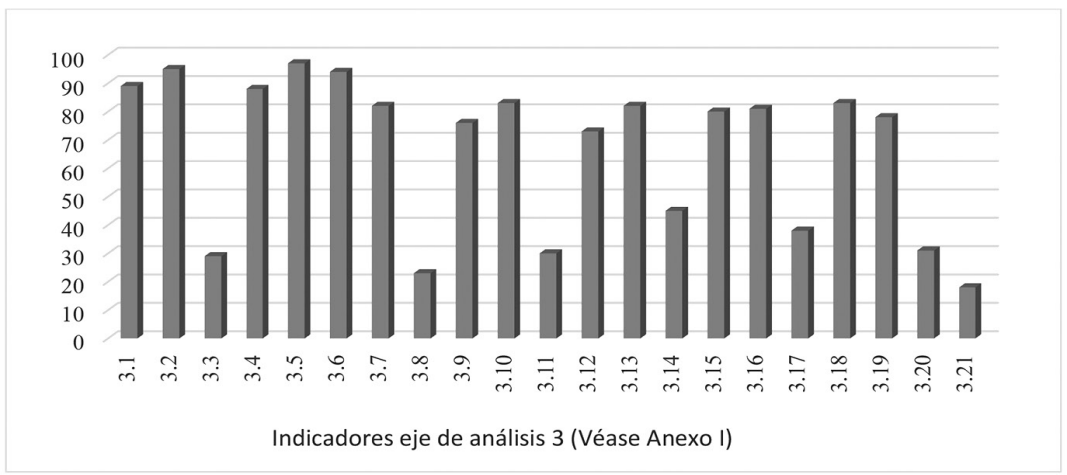

Figura 3. Índices de divulgación de información de cada ítem en el eje de análisis 3 (IDI) Fuente: elaboración propia

En relación al eje de análisis 4, información sobre contratación (servicios y obras), todos los sitios web de los gobiernos tienen un espacio donde publican el perfil del contratante. En España, la nueva Ley 9/2017, de 8 de noviembre, de Contratos del Sector Público, plantea incorporar de forma transversal criterios socialmente responsables a la hora de preparar y ejecutar los contratos públicos, por lo que las administraciones públicas han comenzado 
a elaborar guías y aprobar instrucciones para la implementación de cláusulas socialmente responsables en sus contrataciones. Por este motivo, encontramos obvio dicho resultado. Además, se publica el Plan General de Ordenación Urbana (PGOU) (98\%), se informa sobre la composición y convocatorias de las mesas de contratación (95\%) y se publican los mapas y los planos que se detallan en el PGOU (93 \%). Por su parte, tan sólo $19 \%$ ofrece información relativa a los gastos derivados de urbanismo o informa del presupuesto total de gastos.

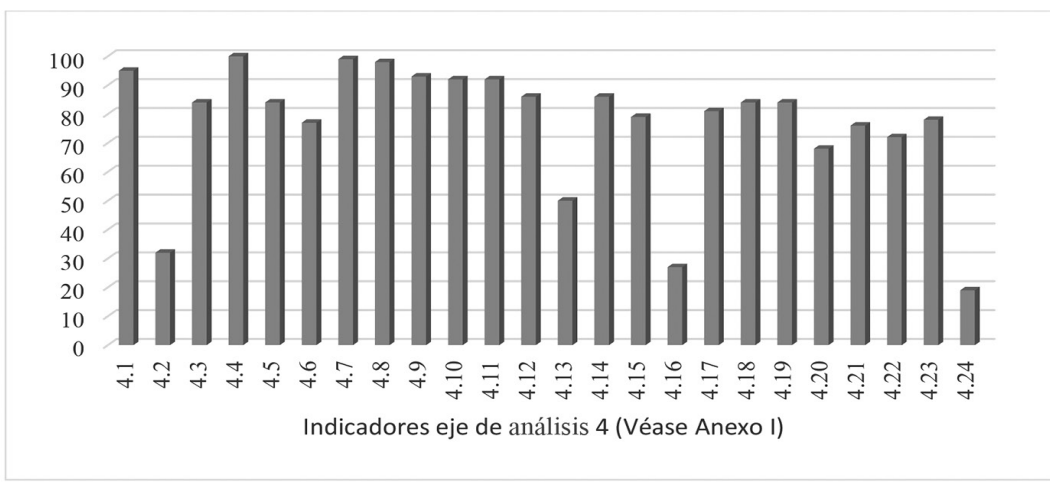

Figura 4. Índices de divulgación de información de cada ítem en el eje de análisis 4 (IDI)

Fuente: elaboración propia

Por último, en la Figura 5, eje correspondiente a información medioambiental, se puede observar que $75 \%$ de los gobiernos locales difunde iniciativas llevadas a cabo para mitigar impactos ambientales, alrededor de $68 \%$ divulga acciones llevadas a cabo para incrementar el ahorro de energía y $64 \%$ informa sobre actuaciones para promover la sensibilidad de los ciudadanos. Sin embargo, son pocos los gobiernos locales que ofrecen información sobre el grado de reducción de las iniciativas $(2 \%)$ y sobre el grado de reducción de ese impacto (2\%). Además, tan sólo $10 \%$ informa sobre el total de gastos e inversiones ambientales.

Del mismo modo, se han calculado los índices de divulgación por cada eje de análisis (Anexo 1) que recogen la aportación de cada eje al índice de divulgación total. En la Figura 6 se observa que el eje más divulgado es el eje de análisis correspondiente a información sobre contratación, servicios y obras $(15.30 \%)$, seguido del tercer y segundo eje de análisis, información económica $(13.29 \%)$ e información social (13.15\%). 


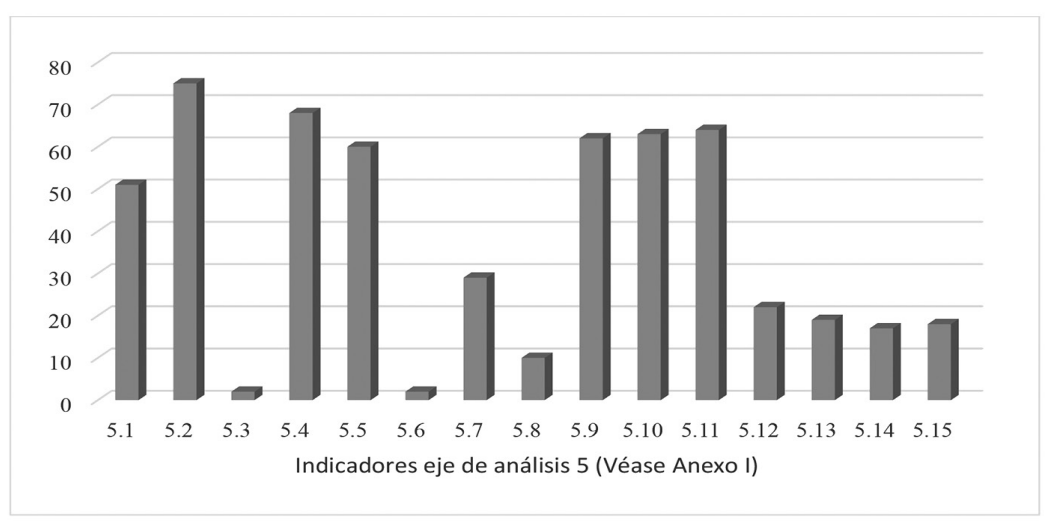

Figura 5. Índices de divulgación de información de cada ítem en el eje de análisis 5 (IDI) Fuente: elaboración propia

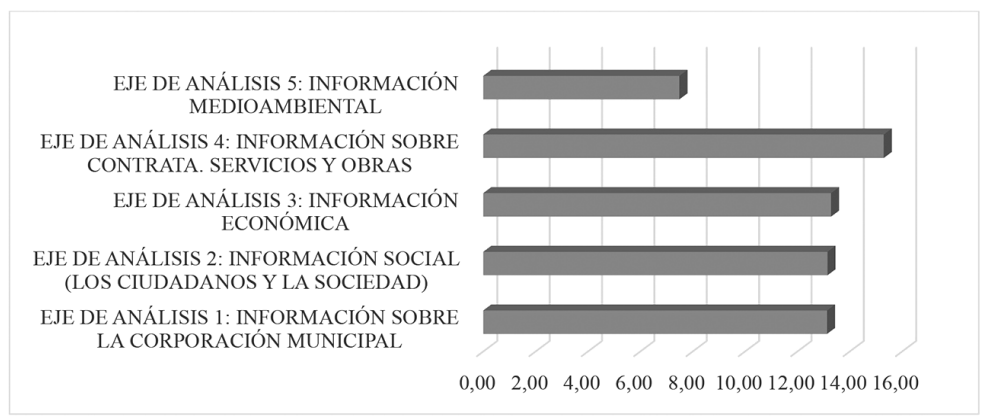

Figura 6. Índices de divulgación por ejes de análisis (IDE)

Fuente: elaboración propia

Además, se ha analizado el índice de divulgación total (IDT), que indica que el conjunto de gobiernos de las capitales de provincia españolas divulga de media $62.36 \%$ de la información total sobre RS (Anexo 2). Por tanto, se observa una alta divulgación de información, por lo que los gobiernos locales tendrán que mejorar sus prácticas de divulgación tan sólo en 37.64 \% para poder alcanzar $100 \%$.

Con base en los índices individuales de cada gobierno local (IDG), en el Anexo 3 se presenta el ranking de las capitales respecto al nivel de información divulgada. Como se puede observar, Madrid encabeza el ranking con un índice de $83.38 \%$, seguido de Zaragoza (80.44 \%), Barcelona (77.58 \%), Alicante $(76.10 \%)$ y Guadalajara (74.42 \%). Las últimas posiciones las ocupan las localidades de Almería (28.74 \%), Pontevedra (31.26 \%) y Jaén ( $40.35 \%$ ). 
Asimismo, puede apreciarse que tan sólo cinco gobiernos, los tres anteriores seguidos de A Coruña y Girona, divulgan por debajo de $50 \%$ de los indicadores propuestos.

Por último, si observamos la información que divulga cada gobierno local en cada eje de análisis (IDGE) (Anexo 3), comprobamos que los gobiernos locales que más información ofrecen sobre la corporación municipal (eje de análisis 1) son Zaragoza, con un índice de divulgación de 17.04 \%, seguido de Barcelona y Alicante, mientras que Madrid (16.43 \%), Valladolid, Pamplona, Albacete y Cáceres (16.07 \% cada uno) son los gobiernos que más información ofrecen sobre información social, los ciudadanos y la sociedad (eje de análisis 2). En cuanto al tercer eje de análisis, información económica, las capitales que más información divulgan son Madrid y Valencia con un índice de divulgación del 19.05 \% y 18.10 \% cada uno. Por su parte, los gobiernos locales de Madrid (19.17 \%) y Salamanca (18.33\%) son los que ocupan las primeras posiciones en divulgación de información referente al cuarto eje de análisis, información sobre contratación, servicios y obras. Las primeras posiciones en el ranking sobre el eje de análisis 5, información medioambiental, las ocupan los gobiernos de Madrid con 14.67 \% y Zaragoza y Alicante con $14 \%$ cada uno.

\section{Discusión}

Los resultados de nuestro estudio, en relación al índice de divulgación total (IDT), que alcanza un valor de $62.36 \%$, mejoran los obtenidos en otros trabajos previos, como los de Nevado y Gallardo (2016), para una muestra de 58 municipios que conforman el Alentejo, en Portugal Continental; los de Navarro, Alcaraz y Ortiz (2010), que analizan una muestra de 55 gobiernos locales españoles, y los de Nevado, Gallardo y Sánchez (2013), para 40 municipios extremeños con población de más de 5000 habitantes. En estos estudios se manifiestan bajos niveles de divulgación, concretamente el $30.69 \%$, el $40.42 \%$ y el $19.19 \%$, respectivamente. Por tanto, y conectando con la primera cuestión planteada en nuestros objetivos, podemos decir que los gobiernos locales de la muestra objeto de estudio divulgan información sobre RS a través de sus páginas web. En relación a la segunda pregunta de investigación, hemos podido constatar, a través del IDT calculado, que las capitales de provincias españolas alcanzan un alto grado de divulgación de información, si lo comparamos con los estudios anteriormente mencionados.

Enlazando con la tercera pregunta de investigación que planteamos, la naturaleza o el tipo de información que se divulga, observamos que los 
gobiernos locales de las capitales de provincias españolas ofrecen más información sobre contratación de servicios y obras públicas. Sin embargo, en el estudio de Nevado y Gallardo (2016), las mayores carencias obedecen a este tipo de información. Este hecho podría justificarse con la entrada en vigor en España, el 9 de marzo, de la nueva normativa en materia de contratación pública, la Ley 9/2017, de 8 de noviembre, de Contratos del Sector Público, por la que se transponen al ordenamiento jurídico español las Directivas del Parlamento Europeo y del Consejo 2014/23/ UE y 2014/24/UE, que plantea incorporar de forma transversal criterios socialmente responsables a la hora de preparar y ejecutar los contratos públicos. Ante esta situación, las administraciones públicas han comenzado a elaborar guías y aprobar instrucciones para la implementación de cláusulas socialmente responsables en sus contrataciones.

Por otro lado, los estudios de Nevado, Gallardo y Sánchez (2013), Navarro, Alcaraz y Ortiz (2010) y Nevado y Gallardo (2016) encontraron mayor divulgación en los aspectos sociales y una escasa divulgación en lo que respecta a la información económica y medioambiental. En los gobiernos locales de nuestra muestra, del mismo modo que los anteriores, ofrecen carencias correspondientes a la información medioambiental; sin embargo, encuentran mayor divulgación en la información económica. Consideramos que uno de los motivos influyentes en la elevada información económica que ofrecen los gobiernos locales de nuestra muestra, en relación con los estudios anteriores, podría ser a la aprobación de la Ley 19/2013, de 9 de diciembre, de Transparencia, Acceso a la Información Pública y Buen Gobierno. Esta ley incrementa y refuerza la transparencia en la actividad pública y reconoce el derecho a la información a los ciudadanos. Como se puede observar en nuestro estudio, existe un alto porcentaje de municipios que divulgan información económica, como las cuentas anuales, los presupuestos y liquidaciones, información sobre ingresos, gastos, en definitiva, información económica clave relativa a aspectos económicos.

\section{CONSIDERACIONES FINALES}

El presente trabajo analiza el grado y el tipo de información divulgada por los gobiernos locales de las provincias españolas a través de sus sitios web, contribuyendo al tema de la RS en el ámbito de las administraciones públicas españolas, ya que no existe una normativa legal que guíe a las mismas en su difusión sistemática. Estas lagunas ponen de manifiesto la necesidad de proponer unas guías de RS a los gobiernos locales de toda España, mejorando así los sistemas de información de cara a su difusión a través de sus webs. 
En respuesta a las preguntas de investigación que planteamos en nuestros objetivos podemos decir, con base en los resultados obtenidos, que los gobiernos locales de las capitales de provincias españolas divulgan información responsable a través de sus páginas web. Al mismo tiempo, hemos podido comprobar que la información que ofrecen es bastante alta, alcanzando un grado de divulgación de $62.36 \%$, tal y como muestra el IDT. Por lo que respecta a la naturaleza de la información divulgada, de los cinco ejes analizados y como se ha comentado previamente, la divulgación de información relacionada sobre la contratación de servicios y obras públicas es la más difundida, seguida de la información económica, en contraposición a la de carácter medioambiental, que constituye la menos divulgada. Esto demuestra un mayor compromiso e implicación por parte de los gobiernos locales españoles con las contrataciones públicas y la dimensión económica de la RS, en relación a los aspectos medioambientales.

Destaca de forma notable el compromiso que muestra Madrid, ya que es la capital que más información divulga de todas las analizadas. Madrid, capital del Estado, constituye la capital de provincia analizada más poblada, la cual ofrece un amplio conjunto de servicios del gobierno nacional para su población y para la población del territorio que la rodea, sirviendo de ejemplo para las demás capitales de provincia. Además de Madrid, los gobiernos locales que más información en materia de RS ofrecen son Zaragoza, Barcelona y Alicante, mientras que Jaén, Pontevedra y Almería son los que menos información divulgan.

En relación con las implicaciones de los resultados obtenidos, consideramos que, para el ámbito académico, nuestro estudio contribuye a la literatura ya existente con una muestra superior, lo que supone una mayor explicación de lo que está sucediendo en relación con la divulgación de información sobre RS en el sector público, debido a la escasez de trabajos en esta materia. En cuanto a sus limitaciones, podemos señalar que se trata de un estudio meramente descriptivo, de corte transversal, por lo que no es posible realizar inferencias. Este motivo nos lleva a plantear una nueva línea de investigación encaminada a analizar los factores explicativos del desarrollo de estas prácticas de divulgación, considerando su evolución en el tiempo. Cabe señalar, además, que el trabajo se centra en el análisis de los sitios web, por lo que no muestra la totalidad de las acciones responsables llevadas a cabo por los gobiernos analizados. Debido a ello se propone, además, una ampliación del estudio que aborde los comportamientos socialmente responsables en otros ámbitos distintos al abordado en nuestro trabajo. 


\section{REFERENCIAS}

AECA (Asociación Española de Contabilidad y Administración de Empresas). 2004. Marco conceptual de la RSC Corporativa. Madrid: Documento 1, Serie Responsabilidad Social Corporativa.

Alcaraz-Quiles, Francisco J., Andrés Navarro-Galera y David Ortiz-Rodríguez. 2015. "Factors determining online sustainability reporting by Local Governments”. International Review of Administrative Sciences 81 (1): 79-109. doi: 10.1177/0020852314541564

Ayuso, M. Dolores y Victoria Martínez. 2005. “Gobierno electrónico. Contenidos y organización de las sedes webs de los parlamentos autonómicos”. Revista española de documentación científica 28 (4): 462-478. doi: https://doi.org/10.3989/redc.2005.v28.i4.175

Bannister, Frank y Regina Connolly. 2011. "The trouble with transparency: A critical review of openness in e government”. Policy E Internet 3 (1): 1-30. doi: 10.2202/1944-2866.1076

Beuren, Ilse M. y Rodrigo Angonese. 2015. "Instruments for determining the disclosure index of accounting information”. Revista Eletrônica de Estratégia e Negócios - REEN 8 (1): 120-144. doi: 10.19177/reen

Brusca, Isabel, Francesca Manes Rossi y Natalia Aversano. 2016. "Online sustainability information in Local Governments in an austerity context: an empirical analysis in Italy and Spain”. Online Information Review 40 (4): 497-514. doi: https://doi.org/10.1108/OIR-05-2015-0161

Carvalho, Luísa C., Dolores Gallardo y M. Teresa Nevado. 2018. "Local municipalities' involvement in promoting entrepreneurship: An analysis of web page orientation to the entrepreneurs in Portuguese municipalities", en Handbook of Research on Entrepreneurial Ecosystems and Social Dynamics in a Globalized World, editado por Luísa C. Carvalho, 1-19. Évora, Portugal: IGI Global. doi: 10.4018/978-1-52253525-6.ch001

Chaín, Celia, Antonio Muñoz y Amalia Más. 2008. "La gestión de información en las sedes webs de los ayuntamientos españoles”. Revista española de documentación cientifica 31 (4): 612-638. doi: 10.3989/redc.2008.4.662

COM (Comisión de las Comunidades Europeas). 2001. Libro Verde. Fomentar un marco europeo para la responsabilidad social de las empresas. Bruselas: Comisión Europea, 366-final. Fecha de consulta: 03/02/2018. http://eur-lex.europa.eu

Cuadrado-Ballesteros, Beatriz, Jennifer Frías-Aceituno y José Martínez-Ferrero. 2014. "The role of media pressure on the disclosure of sustainability information by Local Governments”. Online Information Review 38 (1): 114-135. doi: https:// doi.org/10.1108/OIR-12-2012-0232

Freeman, Edward, Jefrey S. Harrison, Andrew C. Wicks, Bidhan L. Parmar y Simone Colle. 2010. Stakeholder theory. The state of the art. Cambridge: Cambridge University Press. doi: https://doi.org/10.5840/beq20122219

Frías-Aceituno, José V., Maria da Conceição Marques y Lázaro Rodríguez-Ariza. 2013. "Divulgación de información sostenible: ¿se adapta a las expectativas de la sociedad?". Spanish Accounting Review/ Revista de Contabilidad 16 (2): 147-158. doi: https://doi.org/10.1016/j.rcsar.2013.07.004 
Gandía, Juan L. y María C. Archidona. 2008. "Determinants of web site information by Spanish city councils”. Online Information Review 32 (1): 35-57. doi: https:// doi.org/10.1108/14684520810865976

García López, Genaro L. 2007. "El acceso a la información de la administración pública en los Estados Unidos Mexicanos: la regulación del principio de libre información”. Investigación bibliotecológica 21 (43): 107-138. doi: http://dx.doi. org/10.22201/iibi.0187358xp.2007.43.4128

García-Sánchez, Isabel M., José V. Frías-Aceituno y Luis Rodríguez-Domínguez. 2013. "Determinants of Corporate Social Disclosure in Spanish Local Governments". Journal of Cleaner Production 39: 60-72. doi: https://doi.org/10.1016/j. jclepro.2012.08.037

Garde, Raquel, Manuel P. Rodríguez y Antonio M. López. 2015. "Are Australian universities making good use of ICT for CSR reporting?”. Sustainability 7 (11): 14895-14916. doi:10.3390/su71114895

Green, Todd y John Peloza. 2011. "How does corporate social responsibility create value for customers?”. Journal of Consumer Marketing 28 (1): 48-56. doi: https:// doi.org/10.1108/07363761111101949

Hashim, Fathyah, Azlan Amran, Mehran Nejati y Zurih Ismail. 2016. "Examining the determinants of sustainability web-reporting by local authorities in Malaysia”. International Journal of Society Systems Science 8 (2): 155-170. doi: https:// doi.org/10.1504/IJSSS.2016.077012

Kolk, Ans. 2010. "Multinationals and corporate social responsibility". Politeia 26 (98): 138-152.

Kolk, Ans. 2016. "The social responsibility of international business: From ethics and the environment to CSR and sustainable development". Journal of World Business 51 (1): 23-34. doi: 10.1016/j.jwb.2015.08.010

Ley 19/2013, de 9 de diciembre, de Transparencia, Acceso a la Información Pública y Buen Gobierno. Boletín Oficial del Estado 295, España: 97922-97952.

Ley 9/2017, de 8 de noviembre, de Contratos del Sector Público, por la que se transponen al ordenamiento jurídico español las Directivas del Parlamento Europeo y del Consejo 2014/23/UE y 2014/24/UE, de 26 de febrero de 2014. Boletín Oficial del Estado 272, España: 107714-108007.

López, Antonio M. 2010. "Las administraciones públicas ante la Responsabilidad Social”. AECA: Revista de la Asociación Española de Contabilidad y Administración de Empresas, 91, 48-51.

López, Camilo. 2006. El rol del Estado en la Responsabilidad Social de las empresas. Un debate necesario (333.7). Montevideo: Instituto de Comunicación y Desarro1lo, Grupo Uruguay de la Red Puentes.

Ministerio de Trabajo y Asuntos Sociales. 2005. Foro de expertos en Responsabilidad Social de las empresas. Fecha de consulta: 06/08/2018. http://www.um.es/ rscpymes/ficheros/RSC_Informe_Foro_Expertos_Responsabilidad_Social_ Empresarial_Sesiones_I_VI.pdf

Mir, Monir Z., Bikram Chatterjee y Ross Taplin. 2015. "Political competition and environmental reporting: Evidence from New Zealand Local Governments". Asian Review of Accounting 23 (1): 17-38. doi: https://doi.org/10.1108/ARA-02-2014-0027 
Moneva, José M. y Emilio Martín. 2012. “Universidad y Desarrollo sostenible: Análisis de la rendición de cuentas de las universidades públicas desde un enfoque de responsabilidad social”. Revista Iberoamericana de Contabilidad de Gestión 10 (19): $1-18$.

Moura, Ana, Verónica Ribeiro y Sónia Monteiro. 2014. “Divulgação de informação na internet sobre Responsabilidade Social-Evidência empírica nos municípios portugueses”. Trabajo presentado en las XXIV Jornadas Luso-Espanholas de Gestão Científica, Leiria, Portugal, 6 a 8 de febrero.

Mullerat, Ramon. 2010. International corporate social responsibility. The role of corporations in the economic order of the 21st century. London: Kluwer Law International.

Navarro, Andrés, Francisco J. Alcaraz y David Ortiz. 2010. "La divulgación de información sobre responsabilidad corporativa en administraciones públicas: un estudio empírico en gobiernos locales". Revista de Contabilidad 13 (2): 285-314. doi: https://doi.org/10.1016/S1138-4891(10)70019-4

Navarro, Andrés, Mercedes Ruiz, Araceli de los Ríos y Pilar Tirado. 2011. "Responsabilidad social y administración pública local: un análisis del grado de divulgación de información en Reino Unido e Irlanda”. Trabajo presentado en el XVI Congreso AECA, septiembre de 2011. Granada: Asociación Española de Contabilidad y Administración de Empresas.

Navarro, Andrés, Pilar Tirado, Mercedes Ruiz y Araceli de los Ríos. 2015. "Divulgación de información sobre responsabilidad social de los gobiernos locales europeos: El caso de los países nórdicos”. Gestión y Política Pública 24 (1): 229-269.

Navarro Andrés, Francisco J. Alcaraz y David Ortiz. 2016. "Online dissemination of information on sustainability in regional governments. Effects of technological factors". Government Information Quarterly 33 (1): 53-66. doi: https://doi.org/10.1016/j.giq.2015.12.003

Nevado, M. Teresa y Dolores Gallardo. 2016. "Información sobre Responsabilidad Social contenida en las páginas web de los ayuntamientos. Estudio en la región del Alentejo". Revista Española de Documentación Cientifica 39 (4): 150. doi: https://doi.org/10.3989/redc.2016.4.1353

Nevado, M. Teresa, Dolores Gallardo y M. Isabel Sánchez. 2013. "La administración local y su implicación en la creación de una cultura socialmente responsable”. Revista Prisma Social, Revista de Ciencias Sociales, 10, 64-118.

Nevado, M. Teresa, Dolores Gallardo y M. Isabel Sánchez. 2016. “Análisis del grado de divulgación de información sobre responsabilidad social en las webs de los principales municipios extremeños". Auditoría Pública, 67, 77-92.

Nevado, M. Teresa, Patricia Milanés y Jorge M. Prieto. 2018. "Factores determinantes de la divulgación de información sobre emprendimiento por los gobiernos locales españoles". Informação E Sociedade: Estudos 28 (1): 47-60. doi: https://doi. org/10.22478/ufpb.1809-4783.2018v28n1.38225

Pacios, Ana R. 2016. "Universidades transparentes con bibliotecas transparentes". Investigación bibliotecológica 30 (70): 105-128. http://dx.doi.org/10.1016/j.ibbai.2016.10.006

Prado-Lorenzo, José M., Isabel M. García-Sánchez y Beatriz Cuadrado-Ballesteros. 2012. "Sustainable cities: do political factors determine the quality of life?". Journal of Cleaner Production 21 (1): 34-44. doi: https://doi.org/10.1016/j.jclepro.2011.08.021 
Shuler, John A., Paul T. Jaeger y John C. Bertot. 2010. "Implications of harmonizing the future of the federal depository library program within e-government principles and policies". Government Information Quarterly 27 (1): 9-16. doi: https:// doi.org/10.1016/j.giq.2009.09.001

Valor, Carmen e Inmaculada Hurtado. 2009. Las empresas españolas y la responsabilidad social corporativa. La contribución a los objetivos de desarrollo del milenio. Madrid, España: Catarata.

Para citar este texto:

Pache-Durán, María y María Teresa Nevado-Gil. 2019. "Divulgación de información responsable por los gobiernos locales españoles”. Investigación Bibliotecológica: archivonomía, bibliotecología e información 33 (81): 111-134.

http://dx.doi.org/10.22201/iibi.24488321xe.2019.81.58043 


\section{Anexos}

\section{Anexo 1. Índices de divulgación por ítems y por ejes de análisis}

\begin{tabular}{|c|c|}
\hline Eje de análisis 1: información sobre la corporación municipal & IDI \\
\hline $\begin{array}{l}\text { 1.1. Declaración del máximo responsable sobre la relevancia de la } \\
\text { sostenibilidad }\end{array}$ & 56 \\
\hline 1.2. Inclusión en la declaración de prioridades, estrategias & 31 \\
\hline $\begin{array}{l}\text { 1.3. Inclusión en la declaración de desafíos y metas para los próximos } \\
3 / 5 \text { años }\end{array}$ & 17 \\
\hline 1.4. Inclusión de eventos, logros y fracasos durante el periodo de información & 2 \\
\hline 1.5. Especificación de datos biográficos del Alcalde y los concejales & 97 \\
\hline 1.6. Especificación de las direcciones electrónicas del Alcalde y los concejales & 97 \\
\hline 1.7. Información acerca de los sueldos del Alcalde y los concejales & 87 \\
\hline 1.8. Publicación de la relación de puestos de confianza y sus retribuciones & 93 \\
\hline $\begin{array}{l}\text { 1.9. Publicación sobre del Registro de Intereses de Actividades y de bienes } \\
\text { LBRL }\end{array}$ & 91 \\
\hline 1.10. Publicación de la Relación de Puestos de Trabajo & 98 \\
\hline $\begin{array}{l}\text { 1.11. Publicación información sobre procesos selectivos de personal (bases, } \\
\text { adm) }\end{array}$ & 93 \\
\hline 1.12. Especificación de los diferentes Órganos de gobierno y sus funciones & 75 \\
\hline 1.13. Especificación de las direcciones electrónicas de los Órganos de Gobierno & 56 \\
\hline 1.14. Especificación de los datos básicos de organismos y sociedades (con web) & 88 \\
\hline $\begin{array}{l}\text { 1.15. Publicación de la Relación de Puestos de Trabajo de los organismos au- } \\
\text { tónomos }\end{array}$ & 90 \\
\hline $\begin{array}{l}\text { 1.16. Identificación de la persona u órgano responsable de la definición de la } \\
\text { estrategia }\end{array}$ & 26 \\
\hline 1.17. Existencia de representantes de trabajadores & 17 \\
\hline 1.18. Presentación del programa de gobierno & 25 \\
\hline 1.19. Identificación de los compromisos cumplidos del programa de gobierno & 2 \\
\hline 1.20. Información sobre resultados electorales & 22 \\
\hline 1.21. Aprobación y publicación de la Agenda 21 local & 98 \\
\hline 1.22. Órdenes del día previas a los Plenos Municipales & 92 \\
\hline 1.23. Actas de los Plenos Municipales & 91 \\
\hline 1.24. Acuerdos de Plenos & 47 \\
\hline 1.25. Acuerdos de las Juntas de Gobierno & 88 \\
\hline 1.26. Publicación de Ordenanzas y Reglamentos Municipales & 96 \\
\hline 1.27. Existencia y publicación de un "Código de buen gobierno" & 98 \\
\hline Índice de divulgación del eje de análisis 1 (IDE) & 13.13 \\
\hline
\end{tabular}




\begin{tabular}{|c|c|}
\hline Eje de análisis 2: información social (los ciudadanos y la sociedad) & IDI \\
\hline 2.1. Existencia de un mapa de la propia web del gobierno local & 98 \\
\hline 2.2. Existencia de un buscador interno dentro de la página & 100 \\
\hline 2.3. Posibilidad de escuchar la página & 23 \\
\hline 2.4. Existencia de webcams & 0 \\
\hline 2.5. Existencia de wikis compartidas entre departamentos & 0 \\
\hline 2.6. Publicación de datos estadísticos: demográficos, territoriales, etc. & 59 \\
\hline 2.7. Realización de trámites administrativos, permisos, licencias online & 100 \\
\hline $\begin{array}{l}\text { 2.8. Existencia de un Buzón ciudadano o una sección para quejas, } \\
\text { sugerencias }\end{array}$ & 100 \\
\hline 2.9. Información sobre la política de promoción y empleo & 90 \\
\hline 2.10. Identificación sobre los cursos de formación del personal & 81 \\
\hline 2.11. Publicación de la oferta de empleo público & 97 \\
\hline $\begin{array}{l}\text { 2.12. Publicación de horarios y precios de los establecimientos culturales, } \\
\text { deportivos, etc. }\end{array}$ & 60 \\
\hline $\begin{array}{l}\text { 2.13. Publicación de datos sobre el } n^{0} \text { peticiones de ciudadanos resueltas por } \\
\text { silencio administrativo }\end{array}$ & 21 \\
\hline $\begin{array}{l}\text { 2.14. Seguimiento online del estado de tramitación e incidencias del procedi- } \\
\text { miento planteado }\end{array}$ & 98 \\
\hline $\begin{array}{l}\text { 2.15. Información en la web sobre situación y cortes en el tráfico o incidencias } \\
\text { en otros servicios }\end{array}$ & 90 \\
\hline 2.16. Existencia de un espacio para las asociaciones de vecinos & 92 \\
\hline 2.17. Existencia de un servicio de mensajería móvil & 2 \\
\hline 2.18. Existencia de un servicio de chat & 1 \\
\hline 2.19. Existencia radio municipal & 100 \\
\hline 2.20. Publicación de noticias destacadas & 100 \\
\hline 2.21. Información elementos informativos atención ciudadana & 84 \\
\hline 2.22. Existencia de un PUNTOPYME para asesorar empresas & 71 \\
\hline 2.23. Existencia de un enlace para la realización de gestiones sanitarias & 17 \\
\hline 2.24. Existencia y publicación de un Reglamento de Participación ciudadana & 99 \\
\hline 2.25. Existencia de canales de participación como foros, etc. & 80 \\
\hline $\begin{array}{l}\text { 2.26. Posibilidad de sugerencias y participación ciudadana en la elaboración } \\
\text { de los presupuestos }\end{array}$ & 85 \\
\hline 2.27. Publicación de ayudas y subvenciones concedidas & 93 \\
\hline 2.28. Publicación del Salario mínimo local & 0 \\
\hline Índice de divulgación del eje de análisis 2 (IDE) & 13.15 \\
\hline
\end{tabular}




\begin{tabular}{|l|c|}
\hline Eje de análisis 3: información económica & IDI \\
\hline 3.1. Divulgación de cuentas anuales & 89 \\
\hline 3.2. Divulgación de los presupuestos y las liquidaciones & 95 \\
\hline 3.3. Publicación de alegaciones a los presupuestos & 29 \\
\hline 3.4. Publicación de informes periódicos de la ejecución del presupuesto & 88 \\
\hline 3.5. Publicación de modificaciones presupuestarias & 97 \\
\hline 3.6. Publicación de los presupuestos de organismos y sociedades municipales & 94 \\
\hline 3.7. Publicación de informes de auditoría & 82 \\
\hline $\begin{array}{l}\text { 3.8. Existencia de informes gráficos que muestren el desempeño económico } \\
\text { de la Administración }\end{array}$ & 23 \\
\hline $\begin{array}{l}\text { 3.9. Superávit (o déficit) por habitante (resultado presupuestario ajustado/ no } \\
\text { de habitantes) }\end{array}$ & 76 \\
\hline $\begin{array}{l}\text { 3.10. Autonomía fiscal (derechos reconocidos netos de ingresos trib./Der.re- } \\
\text { conocidos netos totales) }\end{array}$ & 83 \\
\hline $\begin{array}{l}\text { 3.11. Publicación de información económica clave, como el PIB, Tasa de Em- } \\
\text { pleo, etc. }\end{array}$ & 30 \\
\hline 3.12. Publicación de las subvenciones recibidas & 73 \\
\hline 3.13. Ingresos fiscales por habitante & 82 \\
\hline 3.14. Gasto por habitante & 45 \\
\hline 3.15. Inversión por habitante & 80 \\
\hline 3.16. Periodo medio de pago & 81 \\
\hline 3.17. Periodo medio de cobro & 38 \\
\hline 3.18. Publicación del importe de la deuda municipal & 83 \\
\hline 3.19. Facilitación de datos acerca de la evolución de la deuda & 31 \\
\hline 3.20. Endeudamiento por habitante & 13.29 \\
\hline 3.21. Endeudamiento relativo & \\
\hline Índice de divulgación del eje de análisis 3 (IDE) & 78 \\
\hline
\end{tabular}

\begin{tabular}{|l|c|}
\hline Eje de análisis 4: información sobre contratación & IDI \\
\hline $\begin{array}{l}\text { 4.1. Información sobre la composición y convocatorias de las mesas } \\
\text { de contratación }\end{array}$ & 95 \\
\hline 4.2. Publicación sobre concursos vigentes de bienes o servicios & 32 \\
\hline $\begin{array}{l}\text { 4.3. Publicación de la resolución de licitaciones, tanto adjudicatarias como } \\
\text { las que no }\end{array}$ & 84 \\
\hline \begin{tabular}{l} 
4.4. Publicación del perfil del contratante \\
\hline $\begin{array}{l}\text { 4.5. Publicación de una lista o cuantía de las operaciones con proveedores + } \\
\text { import. }\end{array}$
\end{tabular} & 84 \\
\hline $\begin{array}{l}\text { 4.6. Publicación de una lista o cuantía de las operaciones con adjudicatarios } \\
\text { +import. }\end{array}$ & 77 \\
\hline
\end{tabular}




\begin{tabular}{|c|c|}
\hline 4.7. Publicación de una lista de empresas contratadas con cargo al FEESL & 0 \\
\hline 4.8. Publicación del Plan General De Ordenación Urbana (PGOU) & 98 \\
\hline 4.9. Publicación de los mapas y planos que se detallan en EL PGOU & 93 \\
\hline 4.10. Publicación de las modificaciones aprobadas en el PGOU & 92 \\
\hline 4.11. Publicación sobre información precisa de los convenios urbanísticos & 92 \\
\hline $\begin{array}{l}\text { 4.12. Publicación de información precisa sobre planes parciales sobre usos } \\
\text { del suelo }\end{array}$ & 86 \\
\hline $\begin{array}{l}\text { 4.13. Publicación de información precisa de la normativa vigente en materia } \\
\text { urbanística }\end{array}$ & 50 \\
\hline $\begin{array}{l}\text { 4.14. Publicación de los proyectos, pliegos y criterios de licitación de obras } \\
\text { públicas }\end{array}$ & 86 \\
\hline $\begin{array}{l}\text { 4.15. Publicación de las modificaciones de los proyectos de obra más impor- } \\
\text { tantes }\end{array}$ & 79 \\
\hline 4.16. Publicación de las licencias de obras concedidas & 27 \\
\hline 4.17. Publicación del listado de empresas que han concurrido en la licitación & 81 \\
\hline $\begin{array}{l}\text { 4.18. Publicación del nombre de las empresas que han realizado obras de ur- } \\
\text { banismo }\end{array}$ & 84 \\
\hline $\begin{array}{l}\text { 4.19. Información precisa sobre cada obra (contratistas, importe presupues- } \\
\text { tado..) }\end{array}$ & 84 \\
\hline $\begin{array}{l}\text { 4.20. Publicación de la fecha concreta prevista para la finalización de las } \\
\text { obras }\end{array}$ & 68 \\
\hline 4.21. Difusión periódica de información sobre obras realizadas & 76 \\
\hline 4.22. Inversión en infraestructuras por habitante/ $n^{\circ}$ habitantes & 72 \\
\hline $\begin{array}{l}\text { 4.23. \% de ingresos derivados de urbanismo/ presupuesto total de los ingre- } \\
\text { sos }\end{array}$ & 78 \\
\hline 4.24. \% gastos derivados de urbanismo/ Presupuesto total de gastos & 19 \\
\hline Índice de divulgación del eje de análisis 4 (IDE) & 15.30 \\
\hline
\end{tabular}

\begin{tabular}{|l|c|}
\hline Eje de análisis 5: información medioambiental & IDI \\
\hline 5.1. Información actualizada sobre situación medioambiental & 51 \\
\hline 5.2. Difusión de iniciativas llevadas a cabo para mitigar impactos ambientales & 75 \\
\hline 5.3. Información sobre el grado de reducción de ese impacto & 2 \\
\hline $\begin{array}{l}\text { 5.4. Difusión de acciones llevadas a cabo para incrementar el ahorro } \\
\text { de energía }\end{array}$ & 68 \\
\hline $\begin{array}{l}\text { 5.5. Información acerca de iniciativas para promocionar consumo eficiente } \\
\text { energía }\end{array}$ & 60 \\
\hline 5.6. Información sobre el grado de reducción de tales iniciativas & 2 \\
\hline 5.7. Información sobre los vertidos y destinos de aguas residuales & 29 \\
\hline 5.8. Información sobre el total de gastos e inversiones ambientales & 1 \\
\hline
\end{tabular}




\begin{tabular}{|l|c|}
\hline 5.9. Información sobre puntos de recogida de basura & 62 \\
\hline 5.10. Información sobre puntos de reciclaje & 63 \\
\hline 5.11. Acciones para promover la sensibilidad de los ciudadanos & 64 \\
\hline 5.12. Información sobre el consumo de energía & 22 \\
\hline 5.13. Información sobre el consumo total de agua & 19 \\
\hline $\begin{array}{l}\text { 5.14. Información sobre sanciones e incumplimiento de la legislación } \\
\text { medioambiental }\end{array}$ & 17 \\
\hline 5.15. Información sobre emisiones totales de gases de efecto invernadero & 18 \\
\hline Índice de divulgación del eje de análisis 5 (IDE) & 7.49 \\
\hline
\end{tabular}

Anexo 2. Índice de divulgación total

Índice de divulgación total (IDT) $(13.13+13.15+13.29+15.30+7.49)$

62.36

Anexo 3. Ranking de los gobiernos locales de las capitales de provincia españolas

\begin{tabular}{|l|c|c|c|c|c|c|}
\hline & IDG & IDGE1 & IDGE2 & IDGE3 & IDGE4 & IDGE5 \\
\hline Madrid & 83.38 & 14.07 & 16.43 & 19.05 & 19.17 & 14.67 \\
\hline Zaragoza & 80.44 & 17.04 & 15.71 & 16.19 & 17.50 & 14.00 \\
\hline Barcelona & 77.58 & 16.67 & 15.36 & 17.14 & 17.08 & 11.33 \\
\hline Alicante & 76.10 & 15.56 & 15.71 & 13.33 & 17.50 & 14.00 \\
\hline Guadalajara & 74.42 & 14.44 & 14.29 & 16.19 & 17.50 & 12.00 \\
\hline León & 73.96 & 14.81 & 15.36 & 14.29 & 17.50 & 12.00 \\
\hline Castellón & 73.63 & 12.96 & 15.71 & 17.62 & 16.67 & 10.67 \\
\hline Valladolid & 73.32 & 14.07 & 16.07 & 14.76 & 17.08 & 11.33 \\
\hline Pamplona & 72.62 & 13.33 & 16.07 & 15.71 & 17.50 & 10.00 \\
\hline Vitoria & 70.95 & 15.19 & 15.00 & 11.43 & 16.67 & 12.67 \\
\hline Zamora & 71.00 & 13.70 & 15.00 & 12.38 & 17.92 & 12.00 \\
\hline San Sebastián & 70.98 & 15.19 & 13.93 & 17.62 & 16.25 & 8.00 \\
\hline $\begin{array}{l}\text { Santa Cruz } \\
\text { de Tenerife }\end{array}$ & 70.74 & 12.59 & 14.64 & 13.33 & 17.50 & 12.67 \\
\hline Murcia & 70.69 & 14.07 & 15.00 & 14.29 & 16.67 & 10.67 \\
\hline Lleida & 70.52 & 11.48 & 13.21 & 15.24 & 17.92 & 12.67 \\
\hline Huesca & 70.25 & 12.96 & 14.64 & 13.81 & 17.50 & 11.33 \\
\hline Ciudad Real & 69.78 & 12.59 & 15.00 & 12.86 & 16.67 & 12.67 \\
\hline Palmas de Gran & 69.58 & 14.44 & 14.64 & 11.90 & 17.92 & 10.67 \\
\hline Canaria & & & & & & \\
\hline
\end{tabular}




\begin{tabular}{|c|c|c|c|c|c|c|}
\hline Oviedo & 68.89 & 11.48 & 14.29 & 17.62 & 14.17 & 11.33 \\
\hline Salamanca & 68.46 & 14.07 & 13.57 & 13.81 & 18.33 & 8.67 \\
\hline Logroño & 68.54 & 14.07 & 13.57 & 13.81 & 17.08 & 10.00 \\
\hline Granada & 67.05 & 13.70 & 13.93 & 13.33 & 15.42 & 10.67 \\
\hline Albacete & 67.18 & 13.70 & 16.07 & 15.24 & 17.50 & 4.67 \\
\hline Córdoba & 64.61 & 14.81 & 13.57 & 10.48 & 17.08 & 8.67 \\
\hline Huelva & 63.93 & 12.59 & 13.93 & 15.24 & 17.50 & 4.67 \\
\hline Santander & 63.10 & 15.19 & 13.21 & 14.29 & 13.75 & 6.67 \\
\hline Málaga & 62.83 & 14.44 & 15.71 & 10.00 & 13.33 & 9.33 \\
\hline Soria & 62.96 & 14.07 & 15.00 & 12.38 & 17.50 & 4.00 \\
\hline Palencia & 62.70 & 14.81 & 14.29 & 14.76 & 17.50 & 1.33 \\
\hline Cádiz & 62.09 & 12.59 & 15.00 & 10.00 & 15.83 & 8.67 \\
\hline Valencia & 62.00 & 12.96 & 15.36 & 18.10 & 12.92 & 2.67 \\
\hline Segovia & 61.65 & 11.11 & 14.29 & 13.33 & 16.25 & 6.67 \\
\hline Cuenca & 61.56 & 13.70 & 13.57 & 14.29 & 16.67 & 3.33 \\
\hline Tarragona & 61.13 & 10.00 & 13.21 & 13.33 & 14.58 & 10.00 \\
\hline Cáceres & 60.56 & 14.07 & 16.07 & 13.33 & 17.08 & 0.00 \\
\hline Toledo & 59.68 & 12.59 & 15.36 & 13.81 & 17.92 & 0.00 \\
\hline $\begin{array}{l}\text { Palma de } \\
\text { Mallorca }\end{array}$ & 59.21 & 14.81 & 14.64 & 10.00 & 17.08 & 2.67 \\
\hline Teruel & 58.31 & 11.11 & 14.64 & 17.14 & 15.42 & 0.00 \\
\hline Lugo & 57.34 & 12.59 & 13.93 & 11.90 & 12.92 & 6.00 \\
\hline Bilbao & 57.00 & 13.33 & 15.00 & 13.33 & 13.33 & 2.00 \\
\hline Ávila & 56.86 & 11.11 & 11.43 & 15.24 & 10.42 & 8.67 \\
\hline Burgos & 56.75 & 12.22 & 13.57 & 10.95 & 16.67 & 3.33 \\
\hline Badajoz & 56.68 & 13.70 & 13.93 & 12.38 & 16.67 & 0.00 \\
\hline Sevilla & 54.85 & 10.74 & 15.00 & 12.86 & 16.25 & 0.00 \\
\hline Ourense & 51.62 & 12.22 & 8.57 & 11.90 & 12.92 & 6.00 \\
\hline Girona & 49.13 & 10.74 & 12.50 & 7.14 & 5.42 & 13.33 \\
\hline A Coruña & 42.61 & 10.74 & 10.36 & 8.10 & 5.42 & 8.00 \\
\hline Jaén & 40.35 & 11.48 & 15.00 & 7.62 & 6.25 & 0.00 \\
\hline Pontevedra & 31.26 & 10.37 & 8.93 & 5.71 & 6.25 & 0.00 \\
\hline Almería & 28.74 & 6.30 & 9.64 & 5.71 & 7.08 & 0.00 \\
\hline
\end{tabular}

